Supporting Information

\title{
Understanding Gas Solubility of Pure Component and Binary Mixtures within Multivalent Ionic Liquids from Molecular Simulations
}

Xiaoyang Liu, Jason E. Bara, C. Heath Turner ${ }^{\star}$,

Department of Chemical and Biological Engineering, The University of Alabama, Tuscaloosa, AL 35487, USA

${ }^{*}$ Corresponding author contact information

hturner@eng.ua.edu

205-348-1733 (ph) 


\section{Methods}

In the current study, a total of nine different ILs are modeled (i.e., 3 cations $x 3$ anions $=9 \mathrm{ILs}$ ), with the chemical structures and respective acronyms summarized in Figure S1. In order to maintain charge neutrality, the following system stoichiometries are used: $\mathrm{A} 1 \mathrm{C} 1\left[\mathrm{NpO}_{2}\right]\left[\mathrm{Bzmim}_{2}\right], \mathrm{A} 1 \mathrm{C2}\left[\mathrm{NpO}_{2}\right]_{3}\left[\mathrm{Bzmim}_{3}\right]_{2}, \mathrm{~A} 1 \mathrm{C} 3\left[\mathrm{NpO}_{2}\right]_{2}\left[\mathrm{Bzmim}_{4}\right], \mathrm{A} 2 \mathrm{C} 1$ $\left[\mathrm{Np}(\mathrm{TfNO})_{2}\right]\left[\mathrm{Bzmim}_{2}\right], \mathrm{A} 2 \mathrm{C} 2\left[\mathrm{~Np}(\mathrm{TfNO})_{2}\right]_{3}\left[\mathrm{Bzmim}_{3}\right]_{2}, \mathrm{~A} 2 \mathrm{C} 3\left[\mathrm{~Np}(\mathrm{TfNO})_{2}\right]_{2}\left[\mathrm{Bzmim}_{4}\right], \mathrm{A} 3 \mathrm{Cl}$ $\left.\left[\mathrm{BzO}_{3}\right]_{2}\left[\mathrm{Bzmim}_{2}\right]_{3}, \mathrm{~A} 3 \mathrm{C} 2\left[\mathrm{BzO}_{3}\right][\mathrm{Bzmim}]_{3}\right]$, and $\mathrm{A} 3 \mathrm{C} 3\left[\mathrm{BzO}_{3}\right]_{4}[\mathrm{Bzmim} 4]_{3}$.

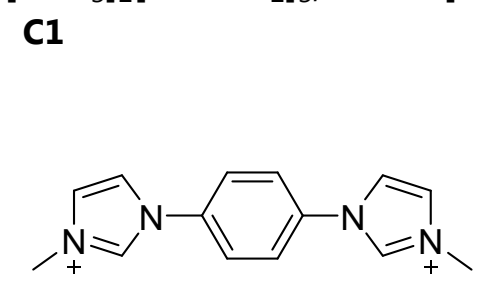

1,1'-(1,4-phenylene)bis(3methylimidazolium)

$\left[\text { Bzmim }_{2}\right]^{2+}$

A1<smiles>O=S(=O)([O-])c1cccc2c(S(=O)(=O)[O-])cccc12</smiles>

naphthalene-1,5-disulfonate $\left[\mathrm{NpO}_{2}\right]^{2-}$
C2<smiles>C[n+]1ccn(-c2cc(-n3cc[n+](C)c3)cc(-n3cc[n+](C)c3)c2)c1</smiles>

1,1',1"-(benzene-1,3,5-triyl)tris(3methylimidazolium)

$\left[\text { Bzmim }_{3}\right]^{3+}$

A2

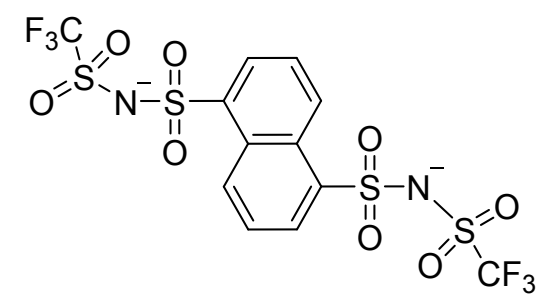

naphthalene-1,5-disulfonylbis(trifluoromethylsulfonylamide)
C3<smiles>C[n+]1ccn(-c2cc(-n3cc[n+](C)c3)c(-n3cc[n+](C)c3)cc2-n2cc[n+](C)c2)c1</smiles>

1,1',1",1'"-(benzene-1,2,4,5tetrayl)tetrakis(3methylimidazolium)

$\left[\text { Bzmim }_{4}\right]^{4+}$
A3<smiles>O=S(=O)([O-])c1cc(S(=O)(=O)[O-])cc(S(=O)(=O)[O-])c1</smiles>

benzene-1,3,5-trisulfonate

$\left[\mathrm{BzO}_{3}\right]^{3-}$

Figure S1-1. Chemical structures of the three cations in the present study $\mathbf{C 1}\left[\mathrm{Bzmim}_{2}\right]^{2+}, \mathbf{C 2}$ $\left[\mathrm{Bzmim}_{3}\right]^{3+}$, and $\mathbf{C} \mathbf{3}\left[\mathrm{Bzmim}_{4}\right]^{4+}$ and the three anions $\mathbf{A} \mathbf{1}\left[\mathrm{NpO}_{2}\right]^{2-}, \mathbf{A} \mathbf{2}\left[\mathrm{Np}(\mathrm{TfNO})_{2}\right]^{2-}$, and $\mathbf{A} \mathbf{3}$ $\left[\mathrm{BzO}_{3}\right]^{3-}$.

\subsection{Force field parameters for the gas molecules}

Table S1. Force field parameter of the gas molecules used in the GCMC/MD simulations.

\begin{tabular}{|c|c|c|c|c|c|c|c|c|c|}
\hline \multirow[b]{2}{*}{ Gas } & \multicolumn{4}{|c|}{ Non-bonded parameters } & \multicolumn{5}{|c|}{ Bonded parameters } \\
\hline & Site & $\varepsilon / k_{B}(\mathrm{~K})$ & $\sigma(\AA)$ & $q(\mathrm{e})$ & Bond & $\begin{array}{l}\text { Length } \\
(\AA)\end{array}$ & Angle & $\theta$ (deg) & $\begin{array}{c}k_{\theta} / k_{B} \\
\left(\mathrm{~K} / \mathrm{rad}^{2}\right)\end{array}$ \\
\hline \multirow{2}{*}{$\mathrm{CO}_{2}$} & $\mathrm{C}$ & 27.0 & 2.80 & 0.700 & $\mathrm{O}-\mathrm{C}$ & 1.16 & $\mathrm{O}-\mathrm{C}-\mathrm{O}$ & 180.0 & Fixed \\
\hline & 0 & 79.0 & 3.05 & -0.350 & & & & & \\
\hline \multirow[t]{2}{*}{$\mathrm{CH}_{4}$} & $C$ & 0.01 & 3.31 & 0 & $\mathrm{H}-\mathrm{C}$ & 1.10 & $\mathrm{H}-\mathrm{C}-\mathrm{H}$ & 107.8 & Fixed \\
\hline & $\mathrm{H}$ & 15.3 & 3.31 & 0 & & & & & \\
\hline \multirow[t]{2}{*}{$\mathrm{N}_{2}$} & $\mathrm{~N}$ & 36.0 & 3.31 & -0.482 & $D-N$ & 0.55 & N-D-N & 180.0 & Fixed \\
\hline & D & 0.0 & 0.00 & 0.964 & & & & & \\
\hline $\mathrm{H}_{2}$ & $\mathrm{H}$ & 12.5 & 2.59 & 0 & $\mathrm{H}-\mathrm{H}$ & 0.74 & & & Fixed \\
\hline \multirow{2}{*}{$\mathrm{SO}_{2}$} & $\mathrm{~S}$ & 73.8 & 3.39 & 0.590 & O-S & 1.43 & $\mathrm{O}-\mathrm{S}-\mathrm{O}$ & 119.3 & 55357 \\
\hline & 0 & 79.0 & 3.05 & -0.295 & & & & & \\
\hline
\end{tabular}




\subsection{Molecular dynamics simulations for pure ILs}

After the geometry optimization of the individual ions, the ion pairs are randomly inserted into the simulation box at a low density by Packmol, ${ }^{1}$ followed by MD simulation in Gromacs 5.0.2 The total number of ions varied, depending upon the system stoichiometry, with a minimum of at least 216 ions of each species. The OPLSAA force field ${ }^{3}$ is used to describe the ion interactions in our system (along with the $1.2{ }^{*} \mathrm{CM} 5$ charges), as assigned by Ligpargen. ${ }^{4}$ The simulations are performed in the isothermal-isobaric (NPT) ensemble, where the Nosé-Hoover thermostat ${ }^{5}$ is used to maintain the temperature, and the pressure is maintained with the Parrinello-Rahman barostat, with time constant of 0.5 and 1 ps, respectively. ${ }^{6-7}$ Following initial relaxation by the steepest-descent algorithm, the equations of motion are integrated with a time step of $1 \mathrm{fs}$ at $300 \mathrm{~K}$ and 1 bar for $10 \mathrm{~ns}$ to equilibrate the system. The production phases are conducted for an additional 10 ns at the same conditions. The LennardJones potential and the electrostatic interactions are calculated with a cutoff distance of $1.2 \mathrm{~nm}$, and the particle mesh Ewald $(\mathrm{PME})^{8}$ method is implemented for long-range electrostatic interaction, with $0.12 \mathrm{~nm}$ of Fourier spacing, while the bonds involving hydrogen atoms are constrained using the LINCS method. ${ }^{9}$ Periodic boundary conditions are implemented in all three dimensions.

\subsection{Gas absorption simulations}

The final frame from the MD simulation stages are used as the initial configurations for the subsequent GCMC simulation stages (temporarily treating the ILs as rigid species). The cross-term Lennard-Jones (LJ) interaction parameters between the ILs and the gas molecules are approximated with the Lorentz-Berthelot mixing rules. In order to increase accelerate equilibration, the systems cycle through multiple alternating stages of GCMC and MD simulations. During the GCMC simulations, the ILs are held rigid but the gas molecules are free to absorb/desorb and move within the IL systems. The GCMC stages are run for $1 \times 10^{7}$ steps (with $33 \%$ insertion, $33 \%$ deletion, $17 \%$ translation, and $17 \%$ rotation).

The MD stages involve full system (ILs + saturated gas molecules) relaxation within the NPT ensemble, with 5 ns for equilibrium and an additional 5 ns for production. These MD simulations are used to analyze the interactions between gas molecules with the ILs, such as the RDFs, average potential energy interactions, and molecular mechanics-based energy decomposition analyses. Some gases display very low solubility ( $<1 \mathrm{~mol}$ ) in the ILs, and the final structures contain no gas molecules. To calculate the RDF and energy analyses, one gas molecule is manually inserted into the IL (at several different locations for statistical analysis). The low gas solubility systems are $\mathrm{CH}_{4}$ and $\mathrm{H}_{2}$ in $\mathrm{A} 1 \mathrm{C} 1$.

The gas solubility in the ILs can be expressed in different ways, depending upon the benchmark or application. Here, we report the "apparent mole fraction" of the gas ( $x^{\prime}$, in units of mol gas $\cdot \mathrm{mol}^{-1} \mathrm{IL}$ ) where $n_{I L}$ represents a mole of the IL. In our case, an IL molecule includes the stoichiometric factors (e.g., one $\mathrm{A} 3 \mathrm{C} 1$ molecule $=$ 
$\left.\left[\mathrm{BzO}_{3}\right]_{2}\left[\mathrm{Bzmim}_{2}\right]_{3}\right)$ :

$$
x^{\prime}=n_{\text {gas }} / n_{\mathrm{IL}}
$$

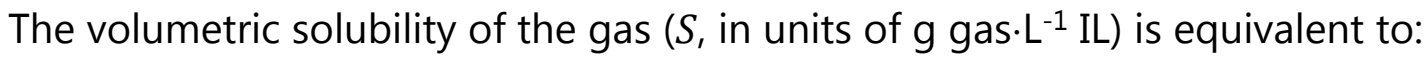

$$
S=x^{\prime} M_{\text {gas }} / V_{m}
$$

where $M_{\text {gas }}$ is the molecular weight of the gas and $V_{m}$ is the molar volume of the neat IL. Here, we focus on the volumetric gas solubility, which provides a more direct context for evaluating the free volume effect.

\subsection{MD/GCMC equilibration tests}

With respect to the MD/GCMC cycles used for equilibration, initial evaluations were used to ensure convergence, and here, we show the largest volumetric solubility IL solvents for each gas (which tend to equilibrate the slowest). For the GCMC simulations, the steps involving the gas absorbates were run for $1 \times 10^{7}$ steps, using 5 $\times 10^{6}$ steps for equilibrium and another $5 \times 10^{6}$ steps for sampling. After the GCMC stage, the last frame is used as the initial configuration for the MD simulations. The $\mathrm{MD}$ simulation relaxation stages are performed for a total of $1 \mathrm{~ns}$, with a time step of $1 \mathrm{fs}$ in the NVT ensemble at the same conditions used in previous MD simulations.

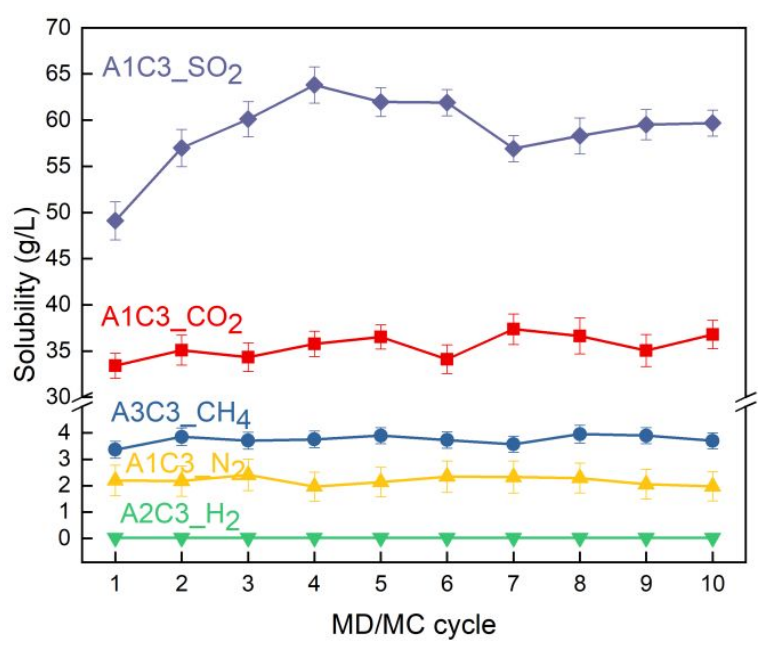

Figure S1-2. Gas solubility in the highest volumetric solubility IL mixtures as a function of the number of alternating MD/GCMC cycles. The systems correspond to $\mathrm{CO}_{2}, \mathrm{~N}_{2}$, and $\mathrm{SO}_{2}$ in $\mathrm{AlC}$, $\mathrm{CH}_{4}$ in $\mathrm{A} 3 \mathrm{C} 3$, and $\mathrm{H}_{2}$ in $\mathrm{A} 2 \mathrm{C} 3$.

\subsection{MD/GCMC cycles for $\mathrm{SO}_{2}$ within ILs}

With respect to the alternating MD/GCMC equilibration and convergence tests, pure $\mathrm{SO}_{2}$ and binary $\mathrm{CO}_{2} / \mathrm{SO}_{2}$ with the mole fractions of $0.25 / 0.75,0.50 / 0.50$, and $0.75 / 0.25$ have been tested for nine different IL systems. The pure absorption of $\mathrm{SO}_{2}$ solubility as a function of MD/GCMC cycles in nine different ILs are shown in Figure S2. The $\mathrm{SO}_{2}$ solubility in $\mathrm{A} 1 \mathrm{C} 3$ changes significantly during the first several MD/MC cycles, but after only a few MD/GCMC cycles $(<10)$, the total $\mathrm{SO}_{2}$ absorption reaches a 
consistent value. With respect to other systems, the $\mathrm{SO}_{2}$ absorption does not change significantly as the number of the MD/GCMC cycles increases. The binary absorption of $\mathrm{CO}_{2} / \mathrm{SO}_{2}$ with a mole faction of $0.50 / 0.50$ is shown as an example in Figure $\mathrm{S} 3$, similar to the behavior for pure $\mathrm{SO}_{2}$ absorption.

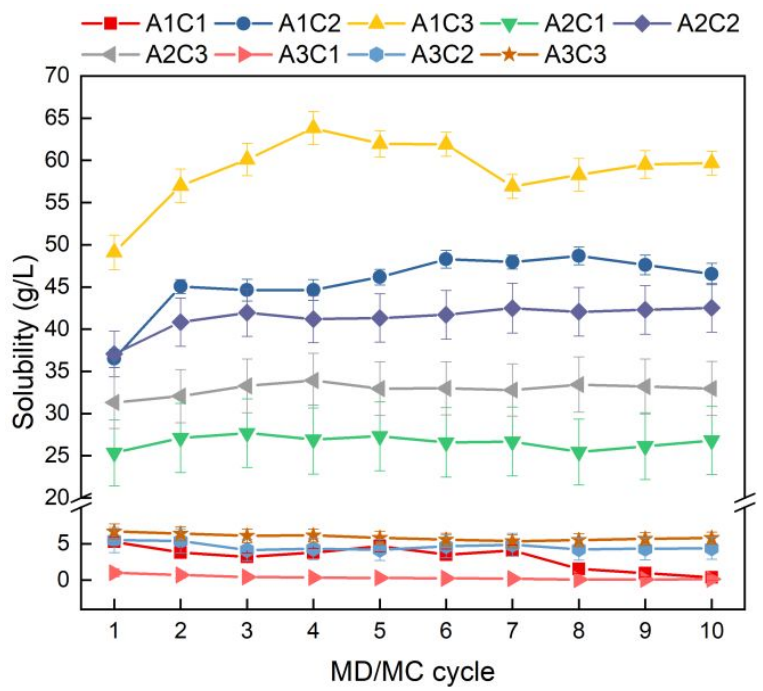

Figure S1-3. Gas solubility of pure $\mathrm{SO}_{2}$ in nine studied IL mixtures as a function of the number of alternating MD/MC cycles.
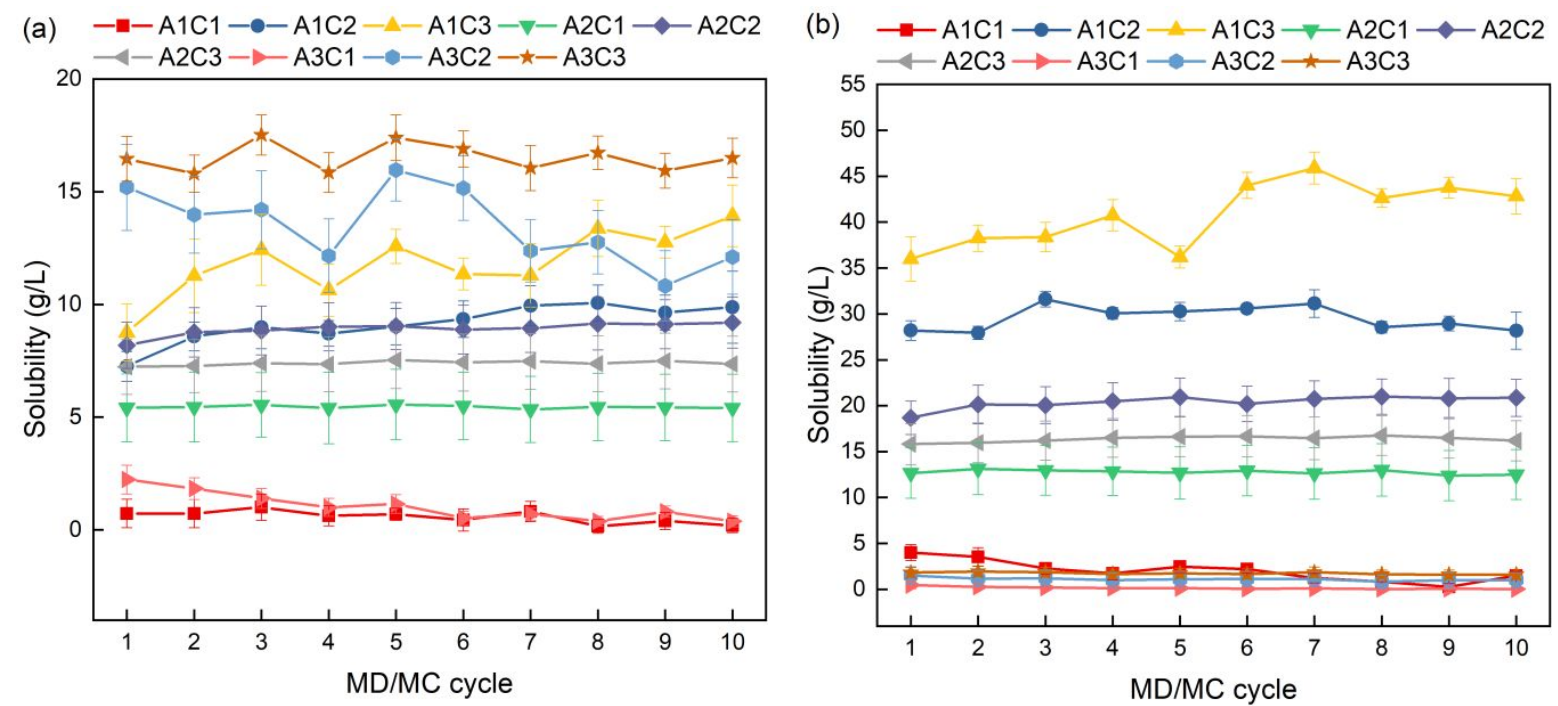

Figure S1-4. Gas solubility of binary absorption of $\mathrm{CO}_{2} / \mathrm{SO}_{2}$ for (a) $\mathrm{CO}_{2}$ and (b) $\mathrm{SO}_{2}$ with a mole fraction of $0.50 / 0.50$ in nine studied IL mixtures as a function of the number of alternating MD/GCMC cycles. 


\section{MD results for pure ILs and GCMC results for single component absorption}

Table S2-1. Summary of the simulated IL physical properties and ESP parameters composed of different ion pairs ( $m=$ cation number and $n=$ anion number) at a temperature of $300 \mathrm{~K}$ and a pressure of 1 bar. All data is taken from our previous work. ${ }^{10}$

\begin{tabular}{|c|c|c|c|c|c|c|c|c|c|c|}
\hline & $\mathrm{m}$ & $\mathrm{n}$ & $\rho$ & $S A$ & $M_{\mathrm{w}}$ & $V_{\mathrm{m}}$ & $F F V$ & $\bar{V}$ & $\bar{V}^{+}$ & $\bar{V}^{-}$ \\
\hline & $\mathrm{mol}$ & $\mathrm{mol}$ & $\mathrm{g} / \mathrm{cm}^{3}$ & $\mathrm{~m}^{2} / \mathrm{cm}^{3}$ & $\mathrm{~g} / \mathrm{mol}$ & $\AA^{3}$ & - & $\mathrm{V}$ & $\mathrm{V}$ & V \\
\hline $\mathrm{A} 1 \mathrm{C} 1$ & 216 & 216 & 1.460 & 16 & 527 & 599 & 0.253 & -0.180 & 1.900 & -2.060 \\
\hline $\mathrm{A} 1 \mathrm{C} 2$ & 432 & 648 & 1.378 & 69 & 1502 & 1809 & 0.310 & -0.050 & 1.690 & -1.715 \\
\hline $\mathrm{A} 1 \mathrm{C} 3$ & 216 & 432 & 1.376 & 140 & 975 & 1177 & 0.321 & -0.050 & 1.740 & -1.760 \\
\hline $\mathrm{A} 2 \mathrm{C} 1$ & 216 & 216 & 1.588 & 103 & 789 & 825 & 0.289 & -0.075 & 1.925 & -1.975 \\
\hline $\mathrm{A} 2 \mathrm{C} 2$ & 432 & 648 & 1.464 & 242 & 2288 & 2595 & 0.360 & 0.060 & 1.620 & -1.580 \\
\hline $\mathrm{A} 3 \mathrm{C} 1$ & 648 & 432 & 1.466 & 85 & 1351 & 1531 & 0.269 & -0.140 & 2.175 & -2.275 \\
\hline $\mathrm{A} 3 \mathrm{C} 2$ & 216 & 216 & 1.411 & 100 & 637 & 749 & 0.319 & 0.070 & 1.925 & -1.860 \\
\hline $\mathrm{A} 3 \mathrm{C} 3$ & 648 & 864 & 1.412 & 181 & 2469 & 2902 & 0.328 & 0.050 & 1.945 & -1.910 \\
\hline
\end{tabular}

Table S2-2. Single component gas volumetric solubility $(\mathrm{g} / \mathrm{L})$ in ILs. The solubility of $\mathrm{SO}_{2}$ is obtained from 10 cycles of MD/GCMC results. The standard deviations reported here are calculated using the block sampling method.

\begin{tabular}{|c|c|c|c|c|c|c|c|c|c|c|c|c|c|c|c|}
\hline \multirow[b]{2}{*}{$\mathrm{A} 1 \mathrm{C} 1$} & \multicolumn{3}{|c|}{$\mathrm{CO}_{2}{ }^{10}$} & \multicolumn{3}{|c|}{$\mathrm{SO}_{2}$} & \multicolumn{3}{|c|}{$\mathrm{N}_{2}$} & \multicolumn{3}{|c|}{$\mathrm{CH}_{4}$} & \multicolumn{3}{|c|}{$\mathrm{H}_{2}$} \\
\hline & 2.95 & \pm & 0.04 & 3.12 & \pm & 0.11 & 0.217 & \pm & 0.008 & 0.119 & \pm & 0.002 & 0.0010 & \pm & 0.0001 \\
\hline A1C2 & 25.64 & \pm & 0.24 & 45.61 & \pm & 0.16 & 1.903 & \pm & 0.017 & 0.397 & \pm & 0.005 & 0.0068 & \pm & 0.0001 \\
\hline A1C3 & 29.69 & \pm & 0.22 & 58.83 & \pm & 0.25 & 2.194 & \pm & 0.008 & 0.739 & \pm & 0.008 & 0.0083 & \pm & 0.0002 \\
\hline $\mathrm{A} 2 \mathrm{C} 1$ & 10.62 & \pm & 0.02 & 26.60 & \pm & 0.07 & 1.488 & \pm & 0.014 & 0.332 & \pm & 0.004 & 0.0184 & \pm & 0.0003 \\
\hline $\mathrm{A} 2 \mathrm{C} 2$ & 16.15 & \pm & 0.03 & 41.35 & \pm & 0.06 & 1.890 & \pm & 0.009 & 0.509 & \pm & 0.005 & 0.0242 & \pm & 0.0002 \\
\hline $\mathrm{A} 2 \mathrm{C} 3$ & 14.24 & \pm & 0.03 & 32.88 & \pm & 0.07 & 1.793 & \pm & 0.010 & 0.490 & \pm & 0.005 & 0.0242 & \pm & 0.0002 \\
\hline $\mathrm{A} 3 \mathrm{C} 2$ & 20.93 & \pm & 0.10 & 4.58 & \pm & 0.09 & 1.169 & \pm & 0.015 & 2.677 & \pm & 0.024 & 0.0079 & \pm & 0.0003 \\
\hline A3C3 & 24.61 & \pm & 0.18 & 5.89 & \pm & 0.06 & 1.543 & \pm & 0.008 & 3.362 & \pm & 0.014 & 0.0089 & \pm & 0.0001 \\
\hline
\end{tabular}

* The text in bold represents the highest solubility of each gas. The solubility of $\mathrm{CO}_{2}$ is taken from previous work. ${ }^{10}$ 
3. GCMC results for binary mixture absorption (the standard deviations reported are calculated using the block sampling method)

Table S3-1. Binary absorption solubility (units of $\mathrm{g} / \mathrm{L}$ ) for $\mathrm{CO}_{2} / \mathrm{SO}_{2}$. The solubilities of $\mathrm{CO}_{2}$ and $\mathrm{SO}_{2}$ are obtained from $10 \mathrm{cycles}$ of $\mathrm{MD} / \mathrm{GCMC}$ results.

\begin{tabular}{|c|c|c|c|c|c|c|c|c|c|c|c|c|c|c|c|c|c|c|}
\hline \multirow[b]{3}{*}{$\mathrm{A} 1 \mathrm{C} 1$} & \multicolumn{9}{|c|}{$y_{\mathrm{CO}_{2}}$} & \multicolumn{9}{|c|}{$y_{\mathrm{SO}_{2}}$} \\
\hline & \multicolumn{3}{|c|}{0.75} & \multicolumn{3}{|c|}{0.50} & \multicolumn{3}{|c|}{0.25} & \multicolumn{3}{|c|}{0.25} & \multicolumn{3}{|c|}{0.50} & \multicolumn{3}{|c|}{0.75} \\
\hline & 1.54 & \pm & 0.05 & 0.69 & \pm & 0.05 & 0.25 & \pm & 0.03 & 2.59 & \pm & 0.08 & 4.05 & \pm & 0.02 & 4.88 & \pm & 0.05 \\
\hline $\mathrm{A} 1 \mathrm{C} 2$ & 13.92 & \pm & 0.06 & 7.38 & \pm & 0.05 & 3.35 & \pm & 0.13 & 19.01 & \pm & 0.23 & 28.22 & \pm & 0.21 & 33.81 & \pm & 0.46 \\
\hline $\mathrm{A} 1 \mathrm{C} 3$ & 15.04 & \pm & 0.19 & 8.89 & \pm & 0.18 & 4.15 & \pm & 0.02 & 24.46 & \pm & 0.17 & 35.55 & \pm & 0.25 & 42.46 & \pm & 0.20 \\
\hline $\mathrm{A} 2 \mathrm{C} 1$ & 8.12 & \pm & 0.02 & 5.38 & \pm & 0.01 & 2.64 & \pm & 0.01 & 6.31 & \pm & 0.03 & 12.67 & \pm & 0.02 & 18.85 & \pm & 0.04 \\
\hline $\mathrm{A} 2 \mathrm{C} 2$ & 12.20 & \pm & 0.01 & 8.23 & \pm & 0.00 & 4.00 & \pm & 0.00 & 9.33 & \pm & 0.01 & 18.71 & \pm & 0.04 & 28.38 & \pm & 0.09 \\
\hline $\mathrm{A} 2 \mathrm{C} 3$ & 10.71 & \pm & 0.03 & 7.21 & \pm & 0.02 & 3.51 & \pm & 0.00 & 7.91 & \pm & 0.02 & 15.87 & \pm & 0.02 & 23.70 & \pm & 0.10 \\
\hline $\mathrm{A} 3 \mathrm{C} 2$ & 18.53 & \pm & 0.05 & 15.20 & \pm & 0.03 & 9.35 & \pm & 0.09 & 0.63 & \pm & 0.01 & 1.47 & \pm & 0.02 & 2.70 & \pm & 0.03 \\
\hline $\mathrm{A} 3 \mathrm{C} 3$ & 20.84 & \pm & 0.04 & 16.25 & \pm & 0.05 & 10.67 & \pm & 0.02 & 0.82 & \pm & 0.00 & 1.80 & \pm & 0.01 & 3.41 & \pm & 0.01 \\
\hline
\end{tabular}

Table S3-2. Solubility selectivity for $\mathrm{CO}_{2}$ from $\mathrm{CO}_{2} / \mathrm{SO}_{2}$ mixtures. The solubility selectivity is obtained from 10 cycles of $\mathrm{MD} / \mathrm{GCMC}$ results.

\begin{tabular}{lcccccccccc}
\hline \multicolumn{3}{c}{$y_{\mathrm{CO}_{2}}=0.75 / y_{\mathrm{SO}_{2}}=0.25$} & \multicolumn{3}{c}{$y_{\mathrm{CO}_{2}}=0.50 / y_{\mathrm{SO}_{2}}=0.50$} & \multicolumn{2}{c}{$y_{\mathrm{CO}_{2}}=0.25 / y_{\mathrm{SO}_{2}}=0.75$} & Ideal \\
\hline $\mathrm{A} 1 \mathrm{C} 1$ & 0.29 & \pm & 0.01 & 0.25 & \pm & 0.02 & 0.22 & \pm & 0.03 & 0.77 \\
$\mathrm{~A} 1 \mathrm{C} 2$ & 0.36 & \pm & 0.00 & 0.38 & \pm & 0.00 & 0.43 & \pm & 0.00 & 1.01 \\
$\mathrm{~A} 1 \mathrm{C} 3$ & 0.30 & \pm & 0.00 & 0.36 & \pm & 0.00 & 0.43 & \pm & 0.00 & 0.92 \\
$\mathrm{~A} 2 \mathrm{C} 1$ & 0.62 & \pm & 0.00 & 0.62 & \pm & 0.00 & 0.61 & \pm & 0.00 & 0.64 \\
$\mathrm{~A} 2 \mathrm{C} 2$ & 0.63 & \pm & 0.00 & 0.64 & \pm & 0.00 & 0.62 & \pm & 0.00 & 0.77 \\
$\mathrm{~A} 2 \mathrm{C} 3$ & 0.66 & \pm & 0.00 & 0.66 & \pm & 0.00 & 0.65 & \pm & 0.00 & 0.74 \\
$\mathrm{~A} 3 \mathrm{C} 1$ & 7.13 & \pm & 0.03 & 7.37 & \pm & 0.06 & 7.36 & \pm & 0.03 & 0.00 \\
$\mathrm{~A} 3 \mathrm{C} 2$ & 14.36 & \pm & 0.04 & 15.04 & \pm & 0.03 & 15.12 & \pm & 0.15 & 5.30 \\
$\mathrm{~A} 3 \mathrm{C} 3$ & 12.35 & \pm & 0.02 & 13.15 & \pm & 0.04 & 13.68 & \pm & 0.03 & 3.28 \\
\hline
\end{tabular}


Table S3-3. Binary absorption solubility (units of $\mathrm{g} / \mathrm{L}$ ) for $\mathrm{CO}_{2} / \mathrm{N}_{2}$.

\begin{tabular}{|c|c|c|c|c|c|c|c|c|c|c|c|c|c|c|c|c|c|c|}
\hline \multirow[b]{3}{*}{$\mathrm{A} 1 \mathrm{C} 1$} & \multicolumn{9}{|c|}{$y_{\mathrm{CO}_{2}}$} & \multicolumn{9}{|c|}{$y_{N_{2}}$} \\
\hline & \multicolumn{3}{|c|}{0.75} & \multicolumn{3}{|c|}{0.50} & \multicolumn{3}{|c|}{0.25} & \multicolumn{3}{|c|}{0.25} & \multicolumn{3}{|c|}{0.50} & \multicolumn{3}{|c|}{0.75} \\
\hline & 2.87 & \pm & 0.04 & 2.42 & \pm & 0.02 & 1.62 & \pm & 0.01 & 0.017 & \pm & 0.002 & 0.040 & \pm & 0.005 & 0.089 & \pm & 0.001 \\
\hline $\mathrm{A} 1 \mathrm{C} 2$ & 24.47 & \pm & 0.08 & 21.12 & \pm & 0.03 & 15.34 & \pm & 0.06 & 0.096 & \pm & 0.002 & 0.242 & \pm & 0.001 & 0.558 & \pm & 0.002 \\
\hline $\mathrm{A} 2 \mathrm{C} 1$ & 8.02 & \pm & 0.02 & 5.47 & \pm & 0.01 & 2.61 & \pm & 0.01 & 0.385 & \pm & 0.005 & 0.746 & \pm & 0.005 & 1.119 & \pm & 0.011 \\
\hline $\mathrm{A} 2 \mathrm{C} 2$ & 12.25 & \pm & 0.04 & 8.19 & \pm & 0.01 & 4.03 & \pm & 0.00 & 0.473 & \pm & 0.001 & 0.950 & \pm & 0.002 & 1.415 & \pm & 0.006 \\
\hline $\mathrm{A} 2 \mathrm{C} 3$ & 10.67 & \pm & 0.01 & 7.13 & \pm & 0.01 & 3.47 & \pm & 0.01 & 0.458 & \pm & 0.001 & 0.904 & \pm & 0.002 & 1.347 & \pm & 0.005 \\
\hline $\mathrm{A} 3 \mathrm{C} 2$ & 18.36 & \pm & 0.04 & 15.16 & \pm & 0.05 & 9.47 & \pm & 0.05 & 0.125 & \pm & 0.002 & 0.279 & \pm & 0.002 & 0.595 & \pm & 0.003 \\
\hline $\mathrm{A} 3 \mathrm{C} 3$ & 20.77 & \pm & 0.06 & 17.08 & \pm & 0.02 & 10.45 & \pm & 0.02 & 0.181 & \pm & 0.001 & 0.440 & \pm & 0.005 & 0.832 & \pm & 0.001 \\
\hline
\end{tabular}

Table S3-4. Solubility selectivity for $\mathrm{CO}_{2}$ from $\mathrm{CO}_{2} / \mathrm{N}_{2}$ mixtures.

\begin{tabular}{lcccccccccc}
\hline \multicolumn{3}{c}{$y_{\mathrm{CO}_{2}}=0.75 / y_{\mathrm{N}_{2}}=0.25$} & \multicolumn{3}{c}{$y_{\mathrm{CO}_{2}}=0.50 / y_{\mathrm{N}_{2}}=0.50$} & \multicolumn{2}{c}{$y_{\mathrm{CO}_{2}}=0.25 / y_{\mathrm{N}_{2}}=0.75$} & Ideal \\
\hline $\mathrm{A} 1 \mathrm{C} 1$ & 36.47 & \pm & 0.48 & 38.09 & \pm & 0.26 & 34.66 & \pm & 0.31 & - \\
$\mathrm{A} 1 \mathrm{C} 2$ & 53.97 & \pm & 0.17 & 55.65 & \pm & 0.07 & 52.50 & \pm & 0.22 & 5.94 \\
$\mathrm{~A} 1 \mathrm{C} 3$ & 35.77 & \pm & 0.15 & 38.98 & \pm & 0.06 & 37.62 & \pm & 0.25 & 6.62 \\
$\mathrm{~A} 2 \mathrm{C} 1$ & 4.43 & \pm & 0.01 & 4.67 & \pm & 0.01 & 4.46 & \pm & 0.01 & - \\
$\mathrm{A} 2 \mathrm{C2}$ & 5.49 & \pm & 0.02 & 5.48 & \pm & 0.01 & 5.44 & \pm & 0.00 & 3.97 \\
$\mathrm{~A} 2 \mathrm{C} 3$ & 4.94 & \pm & 0.00 & 5.02 & \pm & 0.01 & 4.93 & \pm & 0.02 & 4.35 \\
$\mathrm{~A} 3 \mathrm{C} 1$ & 11.53 & \pm & 0.06 & 11.50 & \pm & 0.01 & 11.18 & \pm & 0.05 & - \\
$\mathrm{A} 3 \mathrm{C} 2$ & 31.17 & \pm & 0.06 & 34.57 & \pm & 0.11 & 30.43 & \pm & 0.15 & - \\
$\mathrm{A} 3 \mathrm{C3}$ & 24.40 & \pm & 0.07 & 24.70 & \pm & 0.02 & 23.99 & \pm & 0.06 & 5.88 \\
\hline
\end{tabular}


Table S3-5. Binary absorption solubility (units of $\mathrm{g} / \mathrm{L}$ ) for $\mathrm{CO}_{2} / \mathrm{CH}_{4}$.

\begin{tabular}{|c|c|c|c|c|c|c|c|c|c|c|c|c|c|c|c|c|c|c|}
\hline \multirow[b]{3}{*}{$\mathrm{A} 1 \mathrm{C} 1$} & \multicolumn{9}{|c|}{$y_{\mathrm{CO}_{2}}$} & \multicolumn{9}{|c|}{$y_{\mathrm{CH}_{4}}$} \\
\hline & \multicolumn{3}{|c|}{0.75} & \multicolumn{3}{|c|}{0.50} & \multicolumn{3}{|c|}{0.25} & \multicolumn{3}{|c|}{0.25} & \multicolumn{3}{|c|}{0.50} & \multicolumn{3}{|c|}{0.75} \\
\hline & 2.66 & \pm & 0.02 & 2.32 & \pm & 0.02 & 1.63 & \pm & 0.02 & 0.004 & \pm & 0.002 & 0.015 & \pm & 0.002 & 0.044 & \pm & 0.002 \\
\hline $\mathrm{A} 1 \mathrm{C} 2$ & 23.97 & \pm & 0.08 & 21.69 & \pm & 0.06 & 15.76 & \pm & 0.08 & 0.010 & \pm & 0.002 & 0.034 & \pm & 0.000 & 0.091 & \pm & 0.004 \\
\hline $\mathrm{A} 2 \mathrm{C} 1$ & 8.06 & \pm & 0.01 & 5.32 & \pm & 0.01 & 2.60 & \pm & 0.00 & 0.082 & \pm & 0.001 & 0.165 & \pm & 0.003 & 0.250 & \pm & 0.001 \\
\hline $\mathrm{A} 2 \mathrm{C} 2$ & 12.13 & \pm & 0.02 & 8.21 & \pm & 0.01 & 4.00 & \pm & 0.01 & 0.122 & \pm & 0.000 & 0.253 & \pm & 0.001 & 0.381 & \pm & 0.001 \\
\hline $\mathrm{A} 2 \mathrm{C} 3$ & 10.72 & \pm & 0.02 & 7.17 & \pm & 0.01 & 3.51 & \pm & 0.01 & 0.120 & \pm & 0.001 & 0.245 & \pm & 0.001 & 0.368 & \pm & 0.001 \\
\hline $\mathrm{A} 3 \mathrm{C} 2$ & 18.44 & \pm & 0.02 & 13.53 & \pm & 0.06 & 8.61 & \pm & 0.03 & 0.355 & \pm & 0.003 & 0.899 & \pm & 0.006 & 1.575 & \pm & 0.003 \\
\hline $\mathrm{A} 3 \mathrm{C} 3$ & 20.40 & \pm & 0.05 & 15.77 & \pm & 0.04 & 9.40 & \pm & 0.01 & 0.487 & \pm & 0.003 & 1.120 & \pm & 0.009 & 2.014 & \pm & 0.009 \\
\hline
\end{tabular}

Table S3-6. Solubility selectivity for $\mathrm{CO}_{2}$ from $\mathrm{CO}_{2} / \mathrm{CH}_{4}$ mixtures.

\begin{tabular}{lcccccccccc}
\hline \multicolumn{3}{c}{$y_{\mathrm{CO}_{2}}=0.75 / y_{\mathrm{CH}_{4}}=0.25$} & \multicolumn{3}{c}{$y_{\mathrm{CO}_{2}}=0.50 / y_{\mathrm{CH}_{4}}=0.50$} & \multicolumn{1}{c}{$y_{\mathrm{CO}_{2}}=0.25 / y_{\mathrm{CH}_{4}}=0.75$} & Ideal \\
\hline $\mathrm{A} 1 \mathrm{C} 1$ & 85.53 & \pm & 0.00 & 56.93 & \pm & 0.43 & 40.37 & \pm & 0.47 & - \\
$\mathrm{A} 1 \mathrm{C} 2$ & 296.13 & \pm & 0.01 & 235.75 & \pm & 0.68 & 190.37 & \pm & 0.00 & - \\
$\mathrm{A} 1 \mathrm{C} 3$ & 71.11 & \pm & 0.00 & 70.39 & \pm & 0.19 & 64.87 & \pm & 0.00 & - \\
$\mathrm{A} 2 \mathrm{C} 1$ & 11.90 & \pm & 0.00 & 11.78 & \pm & 0.01 & 11.37 & \pm & 0.01 & - \\
$\mathrm{A} 2 \mathrm{C} 2$ & 12.07 & \pm & 0.00 & 11.84 & \pm & 0.01 & 11.50 & \pm & 0.02 & 8.50 \\
$\mathrm{~A} 2 \mathrm{C} 3$ & 10.84 & \pm & 0.00 & 10.68 & \pm & 0.02 & 10.44 & \pm & 0.02 & - \\
$\mathrm{A} 3 \mathrm{C} 1$ & 3.42 & \pm & 0.00 & 3.31 & \pm & 0.02 & 3.17 & \pm & 0.02 & - \\
$\mathrm{A} 3 \mathrm{C2}$ & 6.31 & \pm & 0.01 & 5.49 & \pm & 0.03 & 5.97 & \pm & 0.02 & 2.62 \\
$\mathrm{~A} 3 \mathrm{C} 3$ & 5.09 & \pm & 0.01 & 5.13 & \pm & 0.01 & 5.10 & \pm & 0.01 & 1.86 \\
\hline
\end{tabular}


Table S3-7. Binary absorption solubility (units of $\mathrm{g} / \mathrm{L}$ ) for $\mathrm{CO}_{2} / \mathrm{H}_{2}$.

\begin{tabular}{|c|c|c|c|c|c|c|c|c|c|c|c|c|c|c|c|c|c|c|}
\hline \multirow[b]{3}{*}{$\mathrm{A} 1 \mathrm{C} 1$} & \multicolumn{9}{|c|}{$y_{\mathrm{CO}_{2}}$} & \multicolumn{9}{|c|}{$y_{\mathrm{H}_{2}}$} \\
\hline & \multicolumn{3}{|c|}{0.75} & \multicolumn{3}{|c|}{0.50} & \multicolumn{3}{|c|}{0.25} & \multicolumn{3}{|c|}{0.25} & \multicolumn{3}{|c|}{0.50} & \multicolumn{3}{|c|}{0.75} \\
\hline & 2.65 & \pm & 0.03 & 2.38 & \pm & 0.02 & 1.61 & \pm & 0.03 & 0.0001 & \pm & 0.0002 & 0.0004 & \pm & 0.0001 & 0.0005 & \pm & 0.0001 \\
\hline $\mathrm{A} 1 \mathrm{C} 2$ & 23.69 & \pm & 0.05 & 21.43 & \pm & 0.05 & 16.38 & \pm & 0.06 & 0.0009 & \pm & 0.0000 & 0.0019 & \pm & 0.0000 & 0.0031 & \pm & 0.0001 \\
\hline $\mathrm{A} 2 \mathrm{C} 1$ & 8.05 & \pm & 0.01 & 5.32 & \pm & 0.01 & 2.61 & \pm & 0.00 & 0.0047 & \pm & 0.0001 & 0.0092 & \pm & 0.0001 & 0.0139 & \pm & 0.0001 \\
\hline $\mathrm{A} 2 \mathrm{C} 2$ & 12.19 & \pm & 0.03 & 8.20 & \pm & 0.01 & 3.99 & \pm & 0.00 & 0.0061 & \pm & 0.0000 & 0.0121 & \pm & 0.0000 & 0.0181 & \pm & 0.0000 \\
\hline $\mathrm{A} 2 \mathrm{C} 3$ & 10.69 & \pm & 0.03 & 7.21 & \pm & 0.01 & 3.50 & \pm & 0.01 & 0.0062 & \pm & 0.0000 & 0.0120 & \pm & 0.0001 & 0.0179 & \pm & 0.0001 \\
\hline $\mathrm{A} 3 \mathrm{C} 2$ & 19.08 & \pm & 0.07 & 15.20 & \pm & 0.04 & 9.99 & \pm & 0.06 & 0.0013 & \pm & 0.0001 & 0.0028 & \pm & 0.0001 & 0.0048 & \pm & 0.0001 \\
\hline A3C3 & 20.96 & \pm & 0.02 & 16.98 & \pm & 0.03 & 10.76 & \pm & 0.02 & 0.0016 & \pm & 0.0000 & 0.0035 & \pm & 0.0001 & 0.0056 & \pm & 0.0001 \\
\hline
\end{tabular}

Table S3-8. Solubility selectivity for $\mathrm{CO}_{2}$ from $\mathrm{CO}_{2} / \mathrm{H}_{2}$ mixtures.

\begin{tabular}{lcccccccccc}
\hline \multicolumn{3}{c}{$y_{\mathrm{CO}_{2}}=0.75 / y_{\mathrm{H}_{2}}=0.25$} & \multicolumn{3}{c}{$y_{\mathrm{CO}_{2}}=0.50 / y_{\mathrm{H}_{2}}=0.50$} & \multicolumn{1}{c}{$y_{\mathrm{CO}_{2}}=0.25 / y_{\mathrm{H}_{2}}=0.75$} & Ideal \\
\hline $\mathrm{A} 1 \mathrm{C} 1$ & 178.10 & \pm & 2.19 & 145.49 & \pm & 1.00 & 215.61 & \pm & 3.38 & - \\
$\mathrm{A} 1 \mathrm{C} 2$ & 209.17 & \pm & 0.47 & 264.25 & \pm & 0.00 & 357.90 & \pm & 0.00 & - \\
$\mathrm{A} 1 \mathrm{C} 3$ & 183.11 & \pm & 0.13 & 215.60 & \pm & 0.00 & 260.69 & \pm & 0.00 & - \\
$\mathrm{A} 2 \mathrm{C} 1$ & 13.01 & \pm & 0.02 & 13.26 & \pm & 0.02 & 12.96 & \pm & 0.02 & - \\
$\mathrm{A} 2 \mathrm{C} 2$ & 15.21 & \pm & 0.03 & 15.53 & \pm & 0.01 & 15.12 & \pm & 0.02 & - \\
$\mathrm{A} 2 \mathrm{C} 3$ & 13.11 & \pm & 0.03 & 13.76 & \pm & 0.02 & 13.43 & \pm & 0.02 & - \\
$\mathrm{A} 3 \mathrm{C} 1$ & 46.32 & \pm & 0.06 & 45.70 & \pm & 0.09 & 47.20 & \pm & 0.27 & - \\
$\mathrm{A} 3 \mathrm{C} 2$ & 108.39 & \pm & 0.37 & 123.27 & \pm & 0.35 & 142.66 & \pm & 0.84 & - \\
$\mathrm{A} 3 \mathrm{C3}$ & 98.88 & \pm & 0.11 & 112.38 & \pm & 0.19 & 131.58 & \pm & 0.28 & - \\
\hline
\end{tabular}




\section{MD results for single component gas molecules within ILs}

\subsection{RDFs of gases with anions and cations}

$\mathrm{SO}_{2}:$

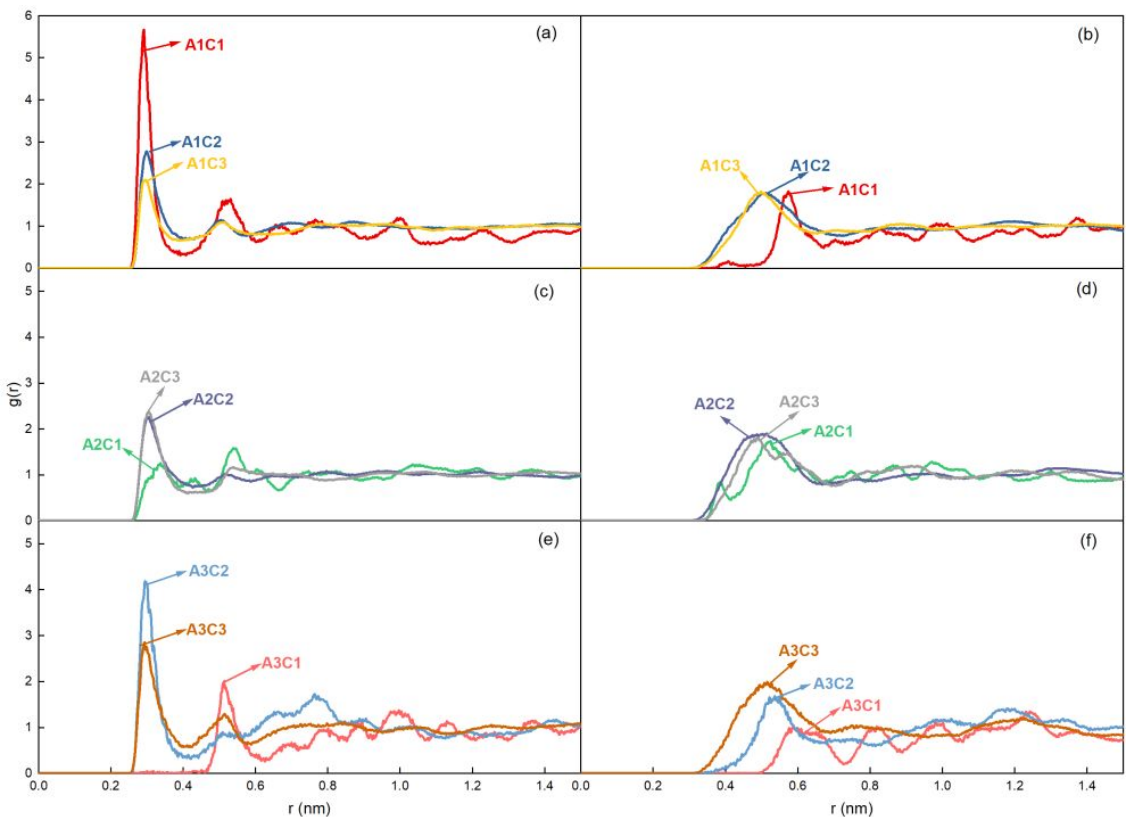

Figure S4-1. Site-site RDFs between the negatively charged anion atoms ( $O$ of $A 1, A 2$ and $A 3$, left panel) and cation atoms (the $\mathrm{N}$ atom linked with $-\mathrm{CH}_{3}$ group in imidazolium ring, right panel) with the sulfur atom in $\mathrm{SO}_{2}$, corresponding to: $(\mathrm{a}, \mathrm{b}) \mathrm{A} 1$-based ILs, $(c, d) \mathrm{A} 2$-based ILs, and (e, f) A3-based ILs.

$\mathbf{N}_{\mathbf{2}}$ :

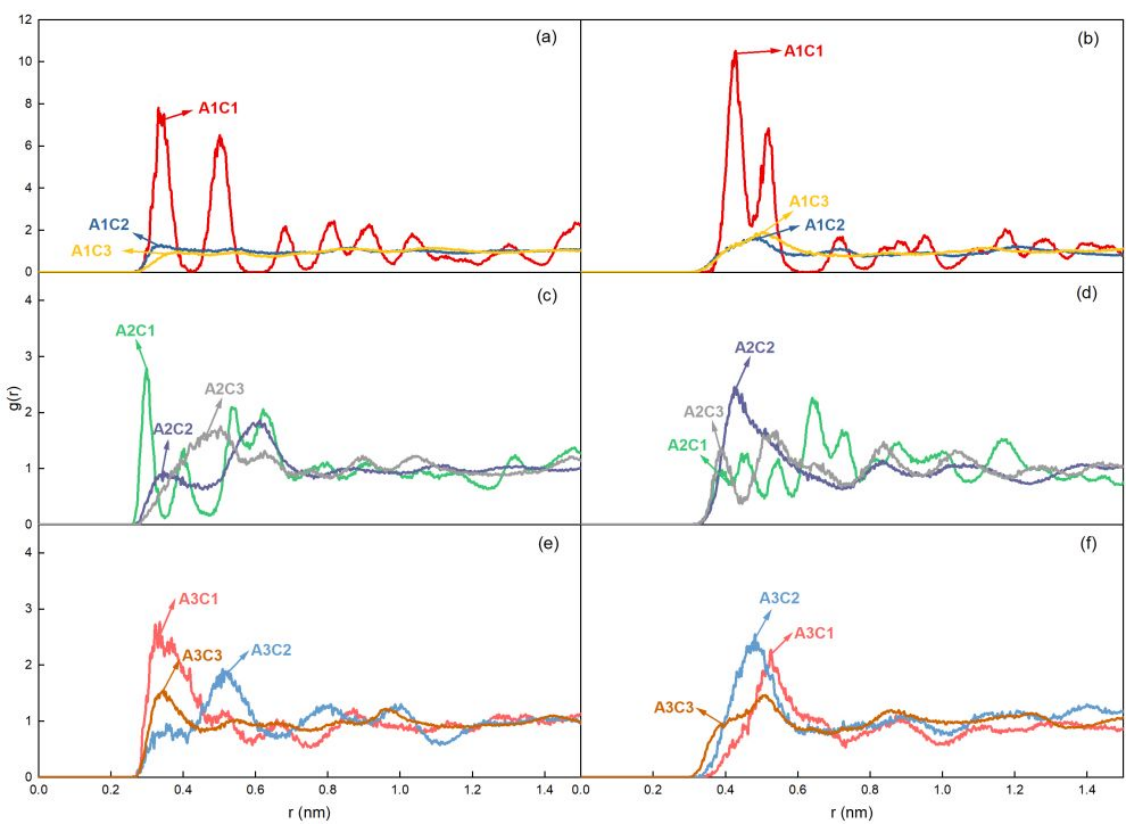

Figure S4-2. Site-site RDFs between the negatively charged anion atoms ( $O$ of $A 1, A 2$ and $A 3$, left panel) and cation atoms (the $\mathrm{N}$ atom linked with $-\mathrm{CH}_{3}$ group in imidazolium ring, right panel) with the positively-charged virtual site in $N_{2}$, corresponding to: $(a, b) A 1$-based ILs, (c, d) A2-based ILs, and (e, f) A3-based ILs. 


\section{$\mathrm{CH}_{4}$ :}

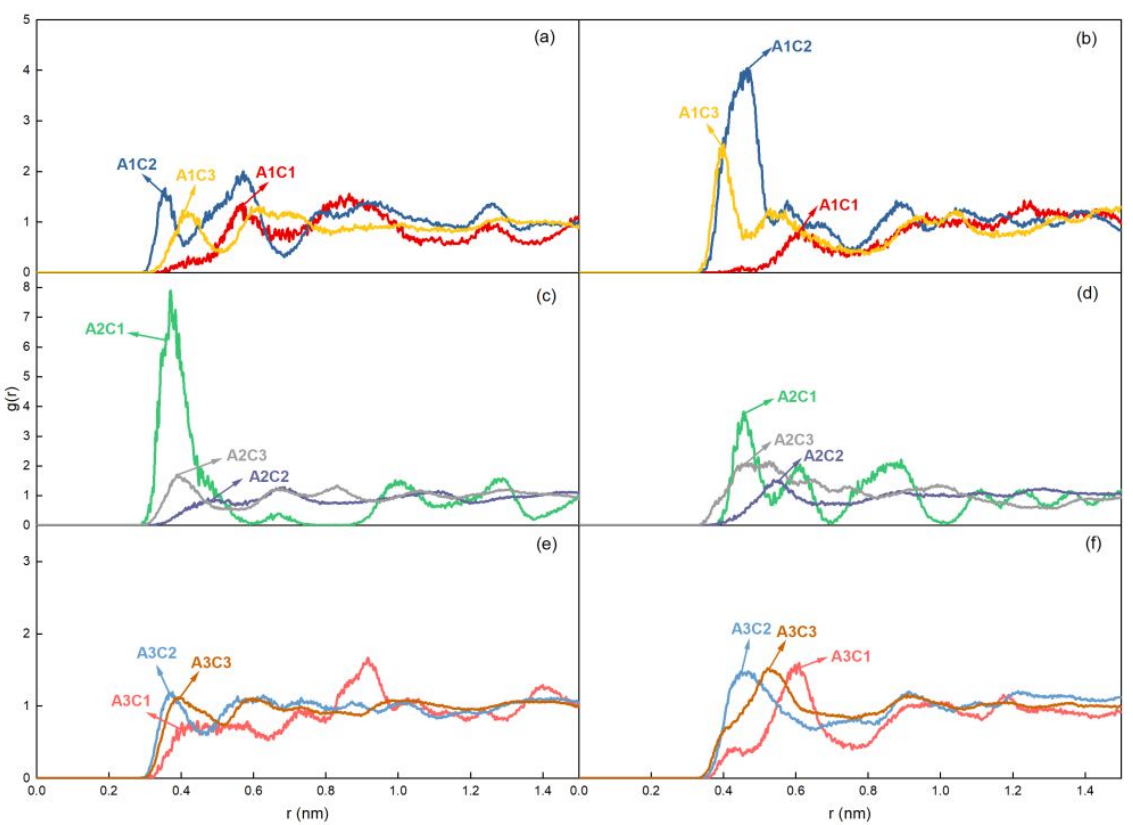

Figure S4-3. Site-site RDFs between the negatively charged anion atoms ( $O$ of $A 1, A 2$ and $A 3$, left panel) and cation atoms (the $\mathrm{N}$ atom linked with $-\mathrm{CH}_{3}$ group in imidazolium ring, right panel) with the carbon atom in $\mathrm{CH}_{4}$, corresponding to: $(\mathrm{a}, \mathrm{b}) \mathrm{A} 1$-based ILs, $(c, d) \mathrm{A} 2$-based ILs, and $(e, f)$ A3-based ILs.

$\mathrm{H}_{\mathbf{2}}$ :

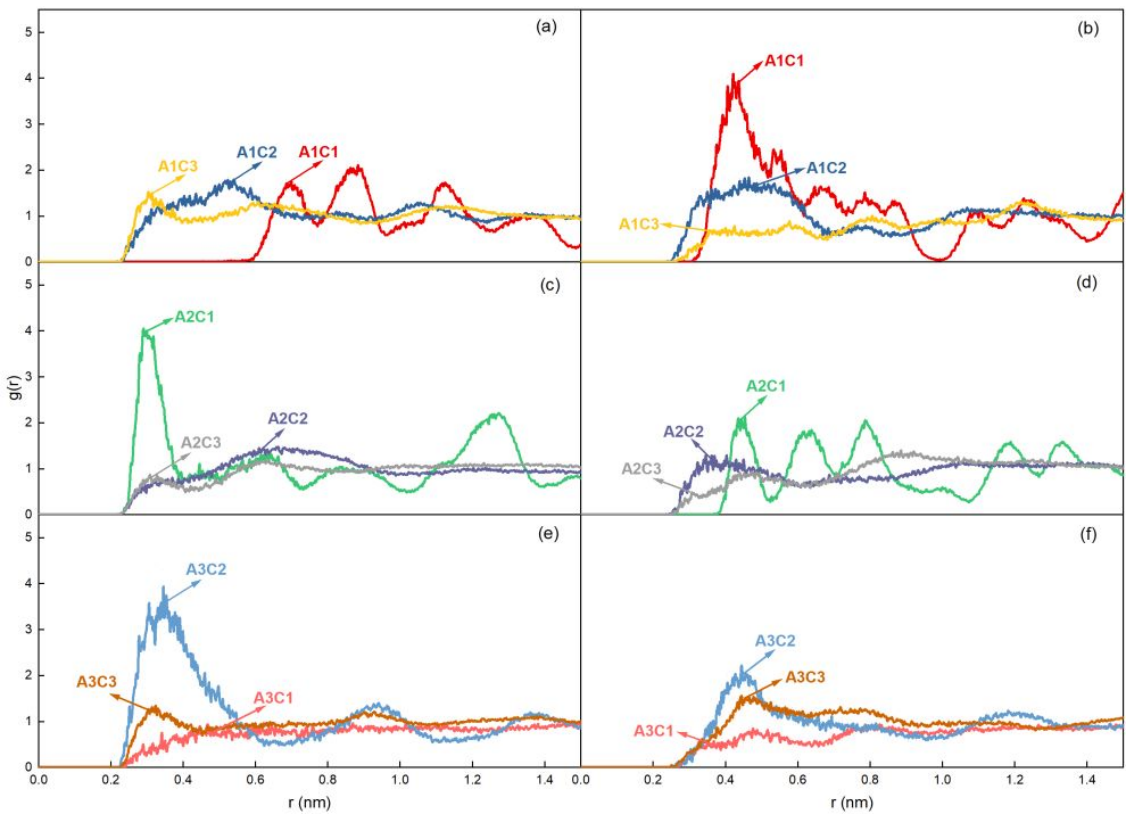

Figure S4-4. Site-site RDFs between the negatively charged anion atoms ( $O$ of $A 1, A 2$ and $A 3$, left panel) and cation atoms (the $\mathrm{N}$ atom linked with $-\mathrm{CH}_{3}$ group in imidazolium ring, right panel) with $\mathrm{H}$ atoms in $\mathrm{H}_{2}$, corresponding to: $(\mathrm{a}, \mathrm{b}) \mathrm{A} 1$-based ILs, $(\mathrm{c}, \mathrm{d}) \mathrm{A} 2$-based ILs, and $(\mathrm{e}, \mathrm{f})$ A3-based ILs. 


\subsection{Average potential energy of interaction between gases with anions and cations at saturation conditions}

Table S4-1. Average potential energy contributions (units of $\mathrm{kcal} / \mathrm{mol}$ ) per $\mathrm{SO}_{2}$ molecule, with $\mathrm{n} \mathrm{\textrm {SO } _ { 2 }}$ molecules absorbed within the different IL solvents after MD/GCMC simulations.

\begin{tabular}{|c|c|c|c|c|c|c|c|c|c|c|c|c|c|c|c|c|c|c|c|c|c|c|c|c|c|c|c|c|}
\hline \multirow{3}{*}{ A1C1 } & \multirow{3}{*}{$\begin{array}{c}\begin{array}{c}\mathrm{nSO}_{2} \\
\mathrm{~mol}\end{array} \\
1\end{array}$} & \multicolumn{9}{|c|}{ Cation.... $\mathrm{SO}_{2}$} & \multicolumn{9}{|c|}{ Anion.... $\mathrm{SO}_{2}$} & \multicolumn{9}{|c|}{$\mathrm{IL} \ldots . . \mathrm{SO}_{2}$} \\
\hline & & \multicolumn{3}{|c|}{$E_{\mathrm{vdW}}$} & \multicolumn{3}{|c|}{$E_{\text {elec }}$} & \multicolumn{3}{|c|}{$E_{\text {tot }}$} & \multicolumn{3}{|c|}{$E_{\mathrm{vdW}}$} & \multicolumn{3}{|c|}{$E_{\text {elec }}$} & \multicolumn{3}{|c|}{$E_{\text {tot }}$} & \multicolumn{3}{|c|}{$E_{\mathrm{vdW}}$} & \multicolumn{3}{|c|}{$E_{\text {elec }}$} & \multicolumn{3}{|c|}{$E_{\text {tot }}$} \\
\hline & & -3.77 & \pm & -3.55 & -35.59 & \pm & 38.96 & -39.36 & \pm & 35.41 & -2.53 & \pm & -0.48 & 43.62 & \pm & -47.33 & 41.09 & \pm & -47.81 & -6.29 & \pm & -4.03 & 8.03 & \pm & -8.37 & 1.73 & \pm & -12.40 \\
\hline A1C2 & 172 & -3.58 & \pm & -3.68 & 3.28 & \pm & -0.74 & -0.30 & \pm & -4.42 & -3.61 & \pm & -3.62 & -12.09 & \pm & -8.18 & -15.70 & \pm & -11.80 & -7.19 & \pm & -7.30 & -8.81 & \pm & -8.92 & -16.00 & \pm & -16.22 \\
\hline A1C3 & 142 & -3.00 & \pm & -3.04 & 4.32 & \pm & 2.08 & 1.32 & \pm & -0.96 & -3.61 & \pm & -3.62 & -12.93 & \pm & -10.76 & -16.54 & \pm & -14.38 & -6.61 & \pm & -6.66 & -8.61 & \pm & -8.68 & -15.22 & \pm & -15.34 \\
\hline $\mathrm{A} 2 \mathrm{C} 1$ & 46 & -9.43 & \pm & 0.22 & -1.37 & \pm & 1.80 & -10.80 & \pm & 1.83 & -3.45 & \pm & 0.19 & -5.25 & \pm & 1.83 & -8.70 & \pm & 1.82 & -12.88 & \pm & 0.28 & -6.62 & \pm & 0.41 & -19.50 & \pm & 0.50 \\
\hline $\mathrm{A} 2 \mathrm{C} 2$ & 219 & -2.79 & \pm & 0.05 & -1.82 & \pm & 1.56 & -4.61 & \pm & 1.57 & -4.52 & \pm & 0.07 & -5.18 & \pm & 1.60 & -9.71 & \pm & 1.60 & -7.32 & \pm & 0.08 & -7.01 & \pm & 0.20 & -14.32 & \pm & 0.20 \\
\hline $\mathrm{A} 2 \mathrm{C} 3$ & 119 & -2.51 & \pm & 0.07 & -1.10 & \pm & 1.83 & -3.62 & \pm & 1.84 & -4.69 & \pm & 0.09 & -6.01 & \pm & 1.82 & -10.70 & \pm & 1.82 & -7.20 & \pm & 0.12 & -7.11 & \pm & 0.30 & -14.31 & \pm & 0.32 \\
\hline АЗС 1 & 1 & -18.57 & \pm & 1.71 & 7.34 & \pm & 10.79 & -11.23 & \pm & 11.17 & -3.34 & \pm & 0.96 & -18.05 & \pm & 10.86 & -21.39 & \pm & 10.89 & -21.91 & \pm & 1.97 & -10.71 & \pm & 1.65 & -32.62 & \pm & 2.08 \\
\hline А 3 C2 & 5 & -4.29 & \pm & 0.39 & -4.92 & \pm & 8.22 & -9.21 & \pm & 8.28 & -2.18 & \pm & 0.46 & -5.83 & \pm & 8.70 & -8.01 & \pm & 8.70 & -6.47 & \pm & 0.58 & -10.74 & \pm & 1.77 & -17.22 & \pm & 1.73 \\
\hline
\end{tabular}

Table S4-2. Average potential energy contributions (units of $\mathrm{kcal} / \mathrm{mol}$ ) per $\mathrm{N}_{2}$ molecule, with $\mathrm{n} \mathrm{N}_{2}$ molecules absorbed within the different IL solvents after MD/GCMC simulations.

\begin{tabular}{|c|c|c|c|c|c|c|c|c|c|c|c|c|c|c|c|c|c|c|c|c|c|c|c|c|c|c|c|c|}
\hline \multirow{3}{*}{$\mathrm{A} 1 \mathrm{C} 1$} & \multirow{3}{*}{$\begin{array}{c}\mathrm{n} \mathrm{N}_{2} \\
\mathrm{~mol}\end{array}$} & \multicolumn{9}{|c|}{ Cation... $\mathrm{N}_{2}$} & \multicolumn{9}{|c|}{ Anion.... $\mathrm{N}_{2}$} & \multicolumn{9}{|c|}{ IL.... $N_{2}$} \\
\hline & & \multicolumn{3}{|c|}{$E_{\mathrm{vdW}}$} & \multicolumn{3}{|c|}{$E_{\text {elec }}$} & \multicolumn{3}{|c|}{$E_{\text {tot }}$} & \multicolumn{3}{|c|}{$E_{\mathrm{vdW}}$} & \multicolumn{3}{|c|}{$E_{\text {elec }}$} & \multicolumn{3}{|c|}{$E_{\text {tot }}$} & \multicolumn{3}{|c|}{$E_{\mathrm{vdW}}$} & \multicolumn{3}{|c|}{$E_{\text {elec }}$} & \multicolumn{3}{|c|}{$E_{\text {tot }}$} \\
\hline & & -9.49 & \pm & 1.07 & -0.40 & \pm & 0.42 & -9.88 & \pm & 1.08 & -1.78 & \pm & 0.73 & -1.52 & \pm & 0.59 & -3.30 & \pm & 0.94 & -11.27 & \pm & 1.21 & -1.92 & \pm & 0.60 & -13.19 & \pm & 1.27 \\
\hline $\mathrm{A} 1 \mathrm{C} 2$ & 16 & -2.00 & \pm & 0.14 & -0.12 & \pm & 0.20 & -2.12 & \pm & 0.23 & -1.74 & \pm & 0.12 & -0.44 & \pm & 0.22 & -2.19 & \pm & 0.25 & -3.75 & \pm & 0.18 & -0.56 & \pm & 0.15 & -4.31 & \pm & 0.22 \\
\hline $\mathrm{A} 1 \mathrm{C} 3$ & 18 & -1.37 & \pm & 0.12 & -0.16 & \pm & 0.17 & -1.53 & \pm & 0.21 & -1.77 & \pm & 0.13 & -0.42 & \pm & 0.19 & -2.19 & \pm & 0.23 & -3.14 & \pm & 0.18 & -0.58 & \pm & 0.16 & -3.72 & \pm & 0.25 \\
\hline $\mathrm{A} 2 \mathrm{C} 1$ & 7 & -4.26 & \pm & 0.32 & -0.28 & \pm & 0.18 & -4.54 & \pm & 0.35 & -1.23 & \pm & 0.41 & -0.58 & \pm & 0.19 & -1.81 & \pm & 0.44 & -5.50 & \pm & 0.50 & -0.85 & \pm & 0.20 & -6.35 & \pm & 0.53 \\
\hline $\mathrm{A} 2 \mathrm{C} 2$ & 18 & -1.31 & \pm & 0.12 & -0.22 & \pm & 0.16 & -1.54 & \pm & 0.19 & -2.17 & \pm & 0.15 & -0.39 & \pm & 0.15 & -2.56 & \pm & 0.22 & -3.48 & \pm & 0.20 & -0.61 & \pm & 0.13 & -4.09 & \pm & 0.24 \\
\hline $\mathrm{A} 2 \mathrm{C} 3$ & 10 & -1.12 & \pm & 0.18 & -0.29 & \pm & 0.21 & -1.41 & \pm & 0.25 & -2.69 & \pm & 0.22 & -0.27 & \pm & 0.19 & -2.97 & \pm & 0.29 & -3.81 & \pm & 0.27 & -0.57 & \pm & 0.21 & -4.38 & \pm & 0.30 \\
\hline $\mathrm{A} 3 \mathrm{C} 1$ & 3 & -3.57 & \pm & 0.77 & -0.22 & \pm & 0.59 & -3.79 & \pm & 0.96 & -0.73 & \pm & 0.23 & -0.39 & \pm & 0.64 & -1.12 & \pm & 0.69 & -4.30 & \pm & 0.84 & -0.61 & \pm & 0.43 & -4.91 & \pm & 1.03 \\
\hline $\mathrm{A} 3 \mathrm{C} 2$ & 4 & -2.34 & \pm & 0.28 & -0.08 & \pm & 0.36 & -2.42 & \pm & 0.44 & -1.48 & \pm & 0.28 & -0.51 & \pm & 0.42 & -1.99 & \pm & 0.52 & -3.82 & \pm & 0.41 & -0.60 & \pm & 0.34 & -4.42 & \pm & 0.55 \\
\hline
\end{tabular}


Table S4-3. Average potential energy contributions (units of $\mathrm{kcal} / \mathrm{mol}$ ) per $\mathrm{CH}_{4}$ molecule, with $\mathrm{n} \mathrm{CH}_{4}$ molecules absorbed within the different IL solvents after MD/GCMC simulations.

\begin{tabular}{|c|c|c|c|c|c|c|c|c|c|c|}
\hline \multirow{3}{*}{$\mathrm{A} 1 \mathrm{C} 1$} & \multirow{3}{*}{$\begin{array}{c}\mathrm{nCH}_{4} \\
\mathrm{~mol}\end{array}$} & \multirow{2}{*}{\multicolumn{3}{|c|}{$\frac{\text { Cation....CH }}{E_{\text {vdW }} / E_{\text {tot }}}$}} & \multirow{2}{*}{\multicolumn{3}{|c|}{$\frac{\text { Anion....CH }}{E_{\mathrm{vdW}} / E_{\text {tot }}}$}} & \multirow{2}{*}{\multicolumn{3}{|c|}{$\frac{\mathrm{IL} \ldots \mathrm{CH}_{4}}{E_{\mathrm{vdW}} / E_{\text {tot }}}$}} \\
\hline & & & & & & & & & & \\
\hline & & -3.07 & \pm & 0.90 & -0.86 & \pm & 0.36 & -3.93 & \pm & 0.99 \\
\hline $\mathrm{A} 1 \mathrm{C} 2$ & 4 & -1.94 & \pm & 0.41 & -1.91 & \pm & 0.30 & -3.85 & \pm & 0.50 \\
\hline $\mathrm{A} 1 \mathrm{C} 3$ & 4 & -0.92 & \pm & 0.33 & -1.04 & \pm & 0.24 & -1.96 & \pm & 0.45 \\
\hline $\mathrm{A} 2 \mathrm{C} 1$ & 1 & -5.39 & \pm & 0.60 & -2.87 & \pm & 0.62 & -8.26 & \pm & 0.91 \\
\hline $\mathrm{A} 2 \mathrm{C} 2$ & 10 & -1.26 & \pm & 0.13 & -2.18 & \pm & 0.21 & -3.44 & \pm & 0.26 \\
\hline $\mathrm{A} 2 \mathrm{C} 3$ & 14 & -1.17 & \pm & 0.13 & -2.31 & \pm & 0.20 & -3.48 & \pm & 0.23 \\
\hline $\mathrm{A} 3 \mathrm{C} 1$ & 6 & -5.24 & \pm & 0.51 & -0.65 & \pm & 0.23 & -5.89 & \pm & 0.60 \\
\hline $\mathrm{A} 3 \mathrm{C} 2$ & 19 & -2.00 & \pm & 0.16 & -1.12 & \pm & 0.11 & -3.12 & \pm & 0.19 \\
\hline $\mathrm{A} 3 \mathrm{C} 3$ & 80 & -2.15 & \pm & 0.08 & -1.61 & \pm & 0.06 & -3.76 & \pm & 0.10 \\
\hline
\end{tabular}

Table S4-4. Average potential energy contributions (units of $\mathrm{kcal} / \mathrm{mol}$ ) per $\mathrm{H}_{2}$ molecule, with $\mathrm{n} \mathrm{H}_{2}$ molecules absorbed within the different IL solvents after MD/GCMC simulations.

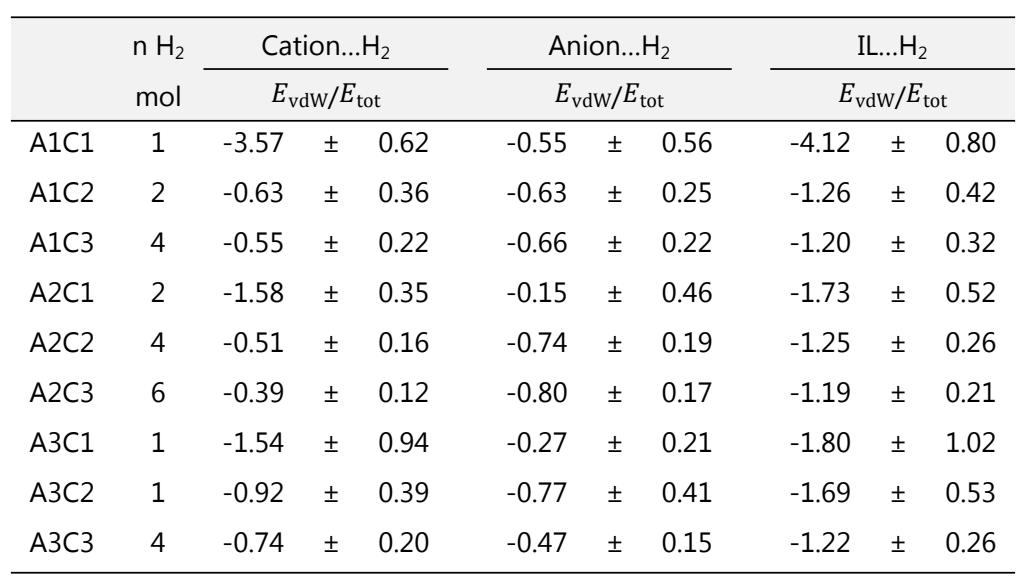




\subsection{Average potential energy of dilute absorption (one gas molecule per system)}

In order to calculate the interaction energy of the ILs with gas molecules, only one gas molecule was present in the simulation, in order to avoid the possibility of any solute-solute interactions. Ten different independent MD simulations were performed (corresponding to ten different initial gas positions) with 1 ns of equilibration in the NPT ensemble and another 1 ns for production.

Table S4-5. Average potential energy contributions (units of $\mathrm{kcal} / \mathrm{mol}$ ) corresponding to one $\mathrm{SO}_{2}$ molecule absorbed within the different IL solvents after MD/GCMC simulations.

\begin{tabular}{|c|c|c|c|c|c|c|c|c|c|c|c|c|c|c|c|c|c|c|c|c|c|c|c|c|c|c|c|}
\hline \multirow[b]{3}{*}{$\mathrm{A} 1 \mathrm{Cl}$} & \multicolumn{9}{|c|}{ Cation....SO $\mathrm{SO}_{2}$} & \multicolumn{9}{|c|}{ Anion.... $\mathrm{SO}_{2}$} & \multicolumn{9}{|c|}{$\mathrm{IL} \ldots \mathrm{SO}_{2}$} \\
\hline & \multicolumn{3}{|c|}{$E_{\mathrm{vdW}}$} & \multicolumn{3}{|c|}{$E_{\text {elec }}$} & \multicolumn{3}{|c|}{$E_{\text {tot }}$} & \multicolumn{3}{|c|}{$E_{\mathrm{vdW}}$} & \multicolumn{3}{|c|}{$E_{\text {elec }}$} & \multicolumn{3}{|c|}{$E_{\text {tot }}$} & \multicolumn{3}{|c|}{$E_{\mathrm{vdW}}$} & \multicolumn{3}{|c|}{$E_{\text {elec }}$} & \multicolumn{3}{|c|}{$E_{\text {tot }}$} \\
\hline & -9.80 & \pm & 3.70 & -24.03 & \pm & 36.75 & -33.83 & \pm & 37.22 & -1.33 & \pm & 1.59 & 20.05 & \pm & 39.08 & 18.71 & \pm & 38.52 & -11.13 & \pm & 4.62 & -3.98 & \pm & 5.22 & -15.12 & \pm & 8.47 \\
\hline $\mathrm{A} 1 \mathrm{C} 2$ & -3.58 & \pm & 1.84 & 5.77 & \pm & 46.88 & 2.19 & \pm & 47.20 & -3.04 & \pm & 1.47 & -13.48 & \pm & 47.57 & -16.52 & \pm & 47.85 & -6.63 & \pm & 2.70 & -7.70 & \pm & 4.33 & -14.33 & \pm & 5.47 \\
\hline $\mathrm{A} 1 \mathrm{C} 3$ & -3.04 & \pm & 1.64 & 8.01 & \pm & 37.51 & 4.97 & \pm & 37.57 & -3.37 & \pm & 1.67 & -15.77 & \pm & 39.48 & -19.15 & \pm & 38.92 & -6.41 & \pm & 2.74 & -7.77 & \pm & 6.28 & -14.18 & \pm & 7.70 \\
\hline $\mathrm{A} 2 \mathrm{C} 1$ & -7.80 & \pm & 2.97 & -5.07 & \pm & 11.87 & -12.87 & \pm & 11.31 & -5.37 & \pm & 1.94 & -2.12 & \pm & 13.69 & -7.49 & \pm & 13.89 & -13.17 & \pm & 3.59 & -7.19 & \pm & 4.34 & -20.36 & \pm & 7.39 \\
\hline $\mathrm{A} 2 \mathrm{C} 2$ & -2.59 & \pm & 0.78 & -4.40 & \pm & 10.18 & -7.00 & \pm & 10.20 & -4.42 & \pm & 1.22 & -2.20 & \pm & 10.60 & -6.62 & \pm & 10.24 & -7.01 & \pm & 1.40 & -6.61 & \pm & 1.92 & -13.62 & \pm & 1.95 \\
\hline $\mathrm{A} 2 \mathrm{C} 3$ & -2.07 & \pm & 0.93 & -12.19 & \pm & 26.58 & -14.26 & \pm & 26.57 & -4.80 & \pm & 1.67 & 7.77 & \pm & 28.70 & 2.97 & \pm & 29.13 & -6.88 & \pm & 1.96 & -4.41 & \pm & 4.67 & -11.29 & \pm & 5.69 \\
\hline $\mathrm{A} 3 \mathrm{C} 1$ & -12.41 & \pm & 5.85 & 13.42 & \pm & 56.66 & 1.00 & \pm & 58.04 & -0.99 & \pm & 1.31 & -27.38 & \pm & 55.20 & -28.36 & \pm & 54.84 & -13.40 & \pm & 5.80 & -13.96 & \pm & 11.45 & -27.36 & \pm & 15.71 \\
\hline $\mathrm{A} 3 \mathrm{C} 2$ & -3.78 & \pm & 1.57 & -15.88 & \pm & 33.39 & -19.66 & \pm & 33.58 & -2.03 & \pm & 1.15 & 7.02 & \pm & 33.53 & 4.99 & \pm & 33.53 & -5.81 & \pm & 2.02 & -8.86 & \pm & 6.14 & -14.67 & \pm & 6.42 \\
\hline $\mathrm{A} 3 \mathrm{C} 3$ & -3.02 & \pm & 1.96 & -6.28 & \pm & 71.49 & -9.30 & \pm & 71.60 & -1.46 & \pm & 1.16 & 0.17 & \pm & 73.39 & -1.30 & \pm & 73.44 & -4.48 & \pm & 2.65 & -6.11 & \pm & 8.03 & -10.59 & \pm & 8.73 \\
\hline
\end{tabular}

Table S4-6. Average potential energy contributions (units of $\mathrm{kcal} / \mathrm{mol}$ ) corresponding to one $\mathrm{N}_{2}$ molecule absorbed within the different IL solvents after MD/GCMC simulations.

\begin{tabular}{|c|c|c|c|c|c|c|c|c|c|c|c|c|c|c|c|c|c|c|c|c|c|c|c|c|c|c|c|}
\hline \multirow[b]{3}{*}{$\mathrm{A} 1 \mathrm{Cl}$} & \multicolumn{9}{|c|}{ Cation... $\mathrm{N}_{2}$} & \multicolumn{9}{|c|}{ Anion.... $\mathrm{N}_{2}$} & \multicolumn{9}{|c|}{ IL.... $\mathrm{N}_{2}$} \\
\hline & \multicolumn{3}{|c|}{$E_{\mathrm{vdW}}$} & \multicolumn{3}{|c|}{$E_{\text {elec }}$} & \multicolumn{3}{|c|}{$E_{\text {tot }}$} & \multicolumn{3}{|c|}{$E_{\mathrm{vdW}}$} & \multicolumn{3}{|c|}{$E_{\text {elec }}$} & \multicolumn{3}{|c|}{$E_{\text {tot }}$} & \multicolumn{3}{|c|}{$E_{\mathrm{vdW}}$} & \multicolumn{3}{|c|}{$E_{\text {elec }}$} & \multicolumn{3}{|c|}{$E_{\text {tot }}$} \\
\hline & -5.80 & \pm & 1.73 & -0.15 & \pm & 0.72 & -5.95 & \pm & 1.93 & -0.63 & \pm & 1.54 & -0.23 & \pm & 0.71 & -0.86 & \pm & 1.62 & -6.42 & \pm & 2.43 & -0.38 & \pm & 0.85 & -6.81 & \pm & 2.52 \\
\hline $\mathrm{A} 1 \mathrm{C} 2$ & -1.29 & \pm & 0.79 & -0.16 & \pm & 1.00 & -1.45 & \pm & 1.24 & -1.72 & \pm & 0.80 & -0.66 & \pm & 1.04 & -2.39 & \pm & 1.42 & -3.02 & \pm & 1.19 & -0.82 & \pm & 0.91 & -3.84 & \pm & 1.51 \\
\hline $\mathrm{A} 1 \mathrm{C} 3$ & -1.25 & \pm & 0.85 & -0.09 & \pm & 0.84 & -1.33 & \pm & 1.19 & -1.77 & \pm & 0.90 & -0.51 & \pm & 0.93 & -2.28 & \pm & 1.41 & -3.02 & \pm & 1.35 & -0.59 & \pm & 0.72 & -3.61 & \pm & 1.74 \\
\hline $\mathrm{A} 2 \mathrm{C} 1$ & -3.19 & \pm & 1.36 & -0.20 & \pm & 0.54 & -3.39 & \pm & 1.43 & -1.60 & \pm & 1.17 & -0.37 & \pm & 0.55 & -1.97 & \pm & 1.34 & -4.79 & \pm & 1.99 & -0.57 & \pm & 0.53 & -5.37 & \pm & 2.17 \\
\hline $\mathrm{A} 2 \mathrm{C} 2$ & -1.20 & \pm & 0.61 & -0.33 & \pm & 0.61 & -1.53 & \pm & 0.85 & -2.20 & \pm & 0.91 & -0.36 & \pm & 0.55 & -2.56 & \pm & 1.13 & -3.40 & \pm & 1.23 & -0.69 & \pm & 0.70 & -4.08 & \pm & 1.51 \\
\hline $\mathrm{A} 2 \mathrm{C} 3$ & -1.28 & \pm & 0.81 & -0.32 & \pm & 0.75 & -1.60 & \pm & 1.12 & -1.61 & \pm & 0.77 & -0.32 & \pm & 0.68 & -1.93 & \pm & 1.04 & -2.89 & \pm & 1.10 & -0.64 & \pm & 0.77 & -3.53 & \pm & 1.44 \\
\hline $\mathrm{A} 3 \mathrm{C} 1$ & -6.47 & \pm & 2.29 & 0.02 & \pm & 0.95 & -6.45 & \pm & 2.52 & -0.81 & \pm & 0.71 & -0.35 & \pm & 1.07 & -1.16 & \pm & 1.35 & -7.28 & \pm & 2.62 & -0.33 & \pm & 0.89 & -7.61 & \pm & 2.99 \\
\hline $\mathrm{A} 3 \mathrm{C} 2$ & -1.56 & \pm & 0.88 & -0.16 & \pm & 0.84 & -1.72 & \pm & 1.20 & -1.35 & \pm & 0.77 & -0.54 & \pm & 0.94 & -1.89 & \pm & 1.33 & -2.91 & \pm & 1.26 & -0.70 & \pm & 0.84 & -3.61 & \pm & 1.64 \\
\hline $\mathrm{A} 3 \mathrm{C} 3$ & -1.62 & \pm & 1.05 & -0.03 & \pm & 1.07 & -1.65 & \pm & 1.56 & -1.46 & \pm & 0.61 & -0.56 & \pm & 1.12 & -2.01 & \pm & 1.28 & -3.08 & \pm & 1.38 & -0.59 & \pm & 0.67 & -3.67 & \pm & 1.63 \\
\hline
\end{tabular}


Table S4-7. Average potential energy contributions (units of $\mathrm{kcal} / \mathrm{mol}$ ) corresponding to one $\mathrm{CH}_{4}$ molecule absorbed within the different $\mathrm{IL}$ solvents after MD/GCMC simulations.

\begin{tabular}{|c|c|c|c|c|c|c|c|c|c|}
\hline \multirow[b]{3}{*}{$\mathrm{A} 1 \mathrm{Cl}$} & \multirow{2}{*}{\multicolumn{3}{|c|}{$\begin{array}{c}\text { Cation....CH } \\
E_{\mathrm{vdW}} / E_{\text {tot }} \\
\end{array}$}} & \multirow{2}{*}{\multicolumn{3}{|c|}{$\frac{\text { Anion....CH }}{E_{\text {vdW }} / E_{\text {tot }}}$}} & \multirow{2}{*}{\multicolumn{3}{|c|}{$\frac{\mathrm{IL} . . . \mathrm{CH}_{4}}{E_{\mathrm{vdW}} / E_{\text {tot }}}$}} \\
\hline & & & & & & & & & \\
\hline & -4.09 & \pm & 1.86 & -0.90 & \pm & 0.84 & -4.99 & \pm & 2.30 \\
\hline $\mathrm{A} 1 \mathrm{C} 2$ & -1.79 & \pm & 0.89 & -2.03 & \pm & 0.88 & -3.82 & \pm & 1.42 \\
\hline $\mathrm{A} 1 \mathrm{C} 3$ & -1.43 & \pm & 0.85 & -1.75 & \pm & 0.84 & -3.18 & \pm & 1.25 \\
\hline $\mathrm{A} 2 \mathrm{C} 1$ & -4.39 & \pm & 1.50 & -0.60 & \pm & 1.66 & -4.99 & \pm & 2.04 \\
\hline $\mathrm{A} 2 \mathrm{C} 2$ & -1.02 & \pm & 0.46 & -1.80 & \pm & 0.81 & -2.82 & \pm & 1.12 \\
\hline $\mathrm{A} 2 \mathrm{C} 3$ & -1.15 & \pm & 0.58 & -2.35 & \pm & 0.87 & -3.51 & \pm & 1.18 \\
\hline $\mathrm{A} 3 \mathrm{C} 1$ & -5.79 & \pm & 1.99 & -0.66 & \pm & 0.96 & -6.45 & \pm & 2.16 \\
\hline $\mathrm{A} 3 \mathrm{C} 2$ & -1.92 & \pm & 0.98 & -1.26 & \pm & 0.78 & -3.18 & \pm & 1.41 \\
\hline АЗС3 & -1.65 & \pm & 1.20 & -1.23 & \pm & 0.83 & -2.88 & \pm & 1.59 \\
\hline
\end{tabular}

Table S4-8. Average potential energy contributions (units of $\mathrm{kcal} / \mathrm{mol}$ ) corresponding to one $\mathrm{H}_{2}$ molecule absorbed within the different $\mathrm{IL}$ solvents after MD/GCMC simulations.

\begin{tabular}{|c|c|c|c|c|c|c|c|c|c|}
\hline \multirow[b]{3}{*}{$\mathrm{A} 1 \mathrm{C} 1$} & \multirow{2}{*}{\multicolumn{3}{|c|}{$\begin{array}{c}\text { Cation.... } \mathrm{H}_{2} \\
E_{\mathrm{vdW}} / E_{\text {tot }}\end{array}$}} & \multirow{2}{*}{\multicolumn{3}{|c|}{$\begin{array}{c}\text { Anion.... } \mathrm{H}_{2} \\
E_{\mathrm{vdW}} / E_{\text {tot }}\end{array}$}} & \multirow{2}{*}{\multicolumn{3}{|c|}{$\frac{\mathrm{IL} \ldots \mathrm{H}_{2}}{E_{\mathrm{vdW}} / E_{\text {tot }}}$}} \\
\hline & & & & & & & & & \\
\hline & -3.05 & \pm & 1.24 & -0.31 & \pm & 0.74 & -3.35 & \pm & 1.35 \\
\hline $\mathrm{A} 1 \mathrm{C} 2$ & -0.72 & \pm & 0.42 & -0.69 & \pm & 0.38 & -1.40 & \pm & 0.53 \\
\hline $\mathrm{A} 1 \mathrm{C} 3$ & -0.61 & \pm & 0.46 & -0.72 & \pm & 0.44 & -1.33 & \pm & 0.64 \\
\hline $\mathrm{A} 2 \mathrm{C} 1$ & -1.42 & \pm & 0.70 & -1.03 & \pm & 0.75 & -2.45 & \pm & 0.91 \\
\hline $\mathrm{A} 2 \mathrm{C} 2$ & -0.57 & \pm & 0.36 & -0.89 & \pm & 0.45 & -1.46 & \pm & 0.57 \\
\hline $\mathrm{A} 2 \mathrm{C} 3$ & -0.42 & \pm & 0.35 & -0.82 & \pm & 0.53 & -1.24 & \pm & 0.62 \\
\hline $\mathrm{A} 3 \mathrm{C} 1$ & -2.50 & \pm & 1.63 & 0.23 & \pm & 1.21 & -2.27 & \pm & 2.41 \\
\hline $\mathrm{A} 3 \mathrm{C} 2$ & -0.83 & \pm & 0.47 & -0.53 & \pm & 0.38 & -1.36 & \pm & 0.68 \\
\hline $\mathrm{A} 3 \mathrm{C} 3$ & -0.63 & \pm & 0.51 & -0.50 & \pm & 0.42 & -1.13 & \pm & 0.73 \\
\hline
\end{tabular}




\section{References:}

1. Martínez, L.; Andrade, R.; Birgin, E. G.; Martínez, J. M., PACKMOL: A Package for Building Initial Configurations for Molecular Dynamics Simulations. J. Comput. Chem. 2009, 30, 2157-2164.

2. Abraham, M. J.; Murtola, T.; Schulz, R.; Páll, S.; Smith, J. C.; Hess, B.; Lindahl, E., GROMACS: High Performance Molecular Simulations Through Multi-Level Parallelism from Laptops to Supercomputers. SoftwareX 2015, 1, 19-25.

3. Robertson, M. J.; Tirado-Rives, J.; Jorgensen, W. L., Improved Peptide and Protein Torsional Energetics with the OPLS-AA Force Field. J. Chem. Theory Comput. 2015, 11, 3499-3509.

4. Dodda, L. S.; Cabeza de Vaca, I.; Tirado-Rives, J.; Jorgensen, W. L., LigParGen Web Server: An Automatic OPLS-AA Parameter Generator for Organic Ligands. Nucleic Acids Res. 2017, 45, W331-W336.

5. Hoover, W. G., Canonical Dynamics: Equilibrium Phase-Space Distributions. Phys. Rev. A 1985, 31, 1695.

6. Parrinello, M.; Rahman, A., Crystal Structure and Pair Potentials: A MolecularDynamics Study. Phys. Rev. Lett. 1980, 45, 1196.

7. Parrinello, M.; Rahman, A., Polymorphic Transitions in Single Crystals: A New Molecular Dynamics Method. J. Appl. Phys. 1981, 52, 7182-7190.

8. Darden, T.; York, D.; Pedersen, L., Particle Mesh Ewald: An $N \cdot \log (N)$ Method for Ewald Sums in Large Systems. J. Chem. Phys. 1993, 98, 10089-10092.

9. Hess, B.; Bekker, H.; Berendsen, H. J.; Fraaije, J. G., LINCS: a Linear Constraint Solver for Molecular Simulations. J. Comput. Chem. 1997, 18, 1463-1472.

10. Liu, X.; O'Harra, K. E.; Bara, J. E.; Turner, C. H., Molecular Insight into the Anion Effect and Free Volume Effect of $\mathrm{CO}_{2}$ Solubility in Multivalent Ionic Liquids. Phys. Chem. Chem. Phys. 2020, 22, 20618-20633. 